\title{
STRA13 Interacts with STAT3 and Modulates Transcription of STAT3-dependent Targets
}

\author{
A. V. Ivanova ${ }^{1 *}$, S. V. Ivanov ${ }^{1}$, X. Zhang ${ }^{2}$, V. N. Ivanov ${ }^{3}$ \\ O. A. Timofeeva ${ }^{4}$ and M. I. Lerman ${ }^{5}$
}

${ }^{1}$ Basic Research Program SAIC-Frederick Inc., National Cancer Institute at Frederick Frederick, MD 21702, USA

${ }^{2}$ Cellular and Molecular Immunology Section Laboratory of Experimental Immunology, Center for Cancer Research, National Cancer Institute at Frederick, Frederick MD 21702, USA

${ }^{3}$ The Ruttenberg Cancer Center Mount Sinai School of Medicine, New York, NY 10029 USA

${ }^{4}$ Laboratory of Comparative Carcinogenesis, Center for Cancer Research, National Cancer Institute at Frederick Frederick, MD 21702, USA

${ }^{5}$ Laboratory of Immunobiology Center for Cancer Research National Cancer Institute at Frederick, Frederick, MD 21702 USA

${ }^{*}$ Corresponding author
STRA13 is a pVHL-dependent bHLH transcription factor up-regulated on the mRNA level in multiple cancer cell lines and implicated recently in the regulation of immune cell homeostasis and autoimmunity. In searching for STRA13-interacting proteins with oncogenic potential by the yeast two-hybrid screening, we identified STAT3 $\beta$ as a STRA13-binding partner. We showed that STRA13 binds predominantly to phosphorylated (active) STAT3 $\alpha$ and $\beta$ isoforms via its HLH and C-terminal regions. We also found that STRA13 was able to activate transcription from STAT-dependent cis-elements. Expression of endogenous STRA13 was shown to be cytokine-inducible, consistent with STRA13 involvement in STAT-dependent transcription regulation. We demonstrated that the STAT3-regulated promoter of the pro-apoptotic Fas gene was activated upon STRA13 over-expression and that co-expression of STRA13 with STAT3 $\beta$ or STAT3 $\alpha$ modulated the transcriptional outcome. Forced expression of STRA13 induced apoptosis, in agreement with the STRA13 activation effect on the Fas promoter. Simultaneous expression of STRA13 and STAT3 $\beta$ resulted in alleviation of the STRA13 pro-apoptotic effect. Thus, for the first time, we identify STRA13 as a STAT3 partner and provide a consistent line of evidence for STRA13 involvement into regulation of apoptosis via the STAT pathways.

(C) 2004 Elsevier Ltd. All rights reserved.

\section{Introduction}

Inactivation of von Hippel-Lindau tumor suppressor protein ( $\mathrm{pVHL}$ ) is critical for the development of certain kidney, CNS, and adrenal tumors. ${ }^{1}$ Recent elucidation of pVHL's pivotal role in regulating expression of numerous genes, via HIF1 $\alpha$ proteolysis, ${ }^{2}$ has helped in delineating

Abbreviations used: STATs, signal transducers and activators of transcription; APRF, acute phase response factor; PV, sodium pervanadate; IL-6, interleukin-6; TAD, transactivation domain.

E-mail address of the corresponding author: ivanova@mail.ncifcrf.gov some of the pVHL biological activities essential for carcinogenesis. It is now clear that while certain pVHL targets, such as VEGF and PDGF, drive tumor-promoted angiogenesis, ${ }^{3,4}$ others, such as GLUT- $1,{ }^{4}$ glycolitic enzymes, ${ }^{5}$ and carbonic anhydrases CA 9 and CA $12^{6,7}$ are responsible for the adjustment of tumor metabolism to hypoxia and acidification (the Warburg effect). We have recently identified a novel pVHL target with an as yet unclear role in tumor progression: the basic helix-loop-helix transcription factor STRA13. ${ }^{6,8}$ The mouse ortholog of human STRA13 was originally characterized as a transcriptional repressor. ${ }^{9}$ Transcriptional repression activity of Stra13 in NIH 3T3 cells was shown to be associated with 
growth arrest and down-regulation of c-Myc. ${ }^{10}$ Molecular mechanisms of Stra13 action involve interaction with members of the general transcriptional machinery, TBP and TFIIB, ${ }^{9}$ and with the transcription factor USF. ${ }^{11}$ Multiple stimuli and mechanisms that regulate the amount of Stra13 in the cell are indicative of its paramount importance for cell function. On the mRNA level, Stra13 is regulated by retinoic acid, cAMP, serum starvation, trichostatin A (a specific histone deacetylase inhibitor) and hypoxia. ${ }^{8-10,12,13}$ On the protein level, it is tightly controlled by the hUBC9-proteasome degradation machinery. 8 There is mounting evidence that cell differentiation is a STRA13 major biological function. Thus, STRA13 expression has been found to be up-regulated in differentiating human embryonic chondrocytes ${ }^{11}$ and in retinoic acid-induced neuronal differentiation of P19 mouse embryonal carcinoma cells. ${ }^{9}$ It was shown recently that hypoxia-induced STRA13 serves as an effector of HIF-1 during inhibition of adipogenesis by hypoxia. ${ }^{14}$ Targeted disruption of Stra13 unexpectedly produced a systemic lupus-like immune disorder highlighting the crucial role of this protein in immune system regulation. ${ }^{15}$ The ineffective elimination of activated $\mathrm{T}$ and B-lymphocytes, as the main result of Stra13 inactivation, led to lymphoid organ hyperplasia, lymphocytic infiltration, and tissue destruction. Another group of researchers characterized Stra13 as a negative regulator of B-lymphocyte activation. ${ }^{16}$

Here, we report on the functional interaction between STRA13 and STAT3. The STAT proteins (signal transducers and activators of transcription) were identified in the last decade as transcription factors that play a pivotal role in the regulation of the immune system, hematopoiesis, neurogenesis and embryogenesis by mediation of virtually all cytokine signaling. JAK-STAT signal transduction events usually require activation of cytoplasmic STAT proteins in response to cytokines through tyrosine phosphorylation, formation of homo- and heterodimers, and entering the nucleus. However, recent experiments revealed the existence of nucleocytoplasmic shuttling of unphosphorylated STATs in the absence of cytokines. ${ }^{17}$ In the nucleus, STAT complexes work coordinately with other transcriptional co-activators and transcription factors to enhance transcriptional initiation. STAT3 was initially identified as acute phase response factor (APRF), an interleukin-6 (IL-6)-inducible protein that binds upon activation to class 2 IL-6 responsive elements. ${ }^{18}$ For the last few years, reports have been accumulating that show STAT3's key role in a variety of different, sometimes opposite, biological processes from cell survival and cell-cycle transition to growth arrest and apoptosis. ${ }^{19}$ The abnormal activation of STAT3 is becoming more frequently associated with unrestricted cell growth and malignant transformation..$^{20,21}$ STAT3 has been classified as an oncogene because constitutively active Stat3 can mediate oncogenic transformation in cell culture and in nude mice. ${ }^{21}$ Two STAT3 protein isoforms, STAT3 $\alpha$ (p92) and STAT3 $\beta$ (p83), have been identified in human and mouse. ${ }^{22-24}$ The mRNA encoding STAT3 $\beta$ has a 50 nucleotide deletion at the $3^{\prime}$ end due to alternative splicing. The resulting protein lacks the C-terminal 55 amino acid residues of STAT $3 \alpha$, which are replaced by seven unique amino acid residues. ${ }^{25}$ The deleted region comprises the transcriptional activation domain. Studies comparing the properties of these two phosphorylated isoforms uncovered a markedly elevated STAT3 $\beta$ DNA-binding activity in comparison with STAT3 $\alpha{ }^{26}$ Biological activity of STAT3 $\beta$ appears to vary in different cells. While in some cases STAT3 $\beta$ was shown to lack transcriptional activity, in other studies it was able to activate promoters of certain genes in a cell type-specific manner. ${ }^{22,23,27}$ The role of the STAT3 $\beta$ isoform in oncogenesis also seems to be cell typespecific. Over-expression of STAT3 $\beta$ efficiently blocked activation of STAT3 signaling and led to growth suppression in melanoma, prostate, ovarian and breast cancers in vitro and in vivo. ${ }^{28-30}$ However, expression of the STAT3 $\beta$ isoform in acute myeloid leukemia patients was found to be associated with shorter overall patient survival. ${ }^{31,32}$ It can be concluded, therefore, that the resulting biological effect of STAT3 transcriptional activity depends largely on the STAT3 $\alpha /$ STAT3 $\beta$ ratio in the cell and on a variety of other transcription factors, co-activators and repressors that may modify the activity of STAT3 proteins.

Our recent findings, linking STRA13 expression with carcinogenesis, encouraged us to pursue its putative role in tumor progression. Because STRA13 protein-partners were poorly investigated, we initiated a study of the potential STRA13 networks using the yeast two-hybrid system. We report here that STRA13 binds phosphorylated STAT3 $\beta$ and STAT3 $\alpha$ isoforms. We further demonstrate that STRA13 activates transcription from STAT-dependent cis-elements and show that co-expression of STAT3 $\beta$ and STRA13 results in suppression of STRA13-dependent transactivation. We also show that expression of the STRA13 gene is cytokine-regulated implicating STRA13 in the JAK-STAT signal transduction pathway. Finally, our data imply STRA13 involvement in the control of apoptosis via STAT3-dependent regulation of the Fas promoter. We suggest, therefore, that inappropriate co-activation of STRA13 and STAT3 may lead to oncogenic transformation.

\section{Results}

\section{STRA13 interacts with STAT3 $\beta$, the short isoform of STAT3}

We searched for STRA13-binding proteins using the yeast two-hybrid system and a human fetal kidney cDNA library. ${ }^{8}$ Out of 186 positive clones, 
which we identified, one represented a full-length $(\sim 3.2 \mathrm{~kb})$ STAT3 $\beta$ cDNA. The specific interaction between STRA13 and STAT3 $\beta$ in yeast was confirmed by co-transformation of both the BD-STRA13 and AD-STAT3 constructs into yeast cells resulting in reconstitution of GAL4 transcription activity (data not shown). To verify the interaction between pSTRA13 and pSTAT3 $\beta$ in mammalian cells, we fused their $\mathrm{N}$ termini with FLAG-tag and HA-tag, respectively, in pCMV2 and pCMV-HA expression vectors. Co-immunoprecipitation confirmed that the STAT3 $\beta-\mathrm{HA}$ protein specifically interacted with the STRA13FLAG protein (Figure 1(A), line 2). No precipitation was observed when the empty pCMV2 vector was co-transfected with STAT3 $\beta$-HA vector (Figure 1A, line 1).

\section{STAT3 $\beta$ associates with the HLH and the C-terminal regions of STRA13}

To define the STRA13 domain(s) responsible for binding to STAT3 $\beta$ we used a set of STRA13 FLAG-fused deletion constructs (Figure 1A, upper part). The STAT3-HA and each of the STRA13 deletion variants were transiently cotransfected into 293T cells. Twenty-four hours after transfection, the cells were treated with the proteasome inhibitor ALLnL and subjected to co-immunoprecipitation analysis. ALLnL treatment was performed to stabilize the truncated proteins, since we have shown that truncation of either STRA13 terminus leads to a sharp decrease in the protein half-life. ${ }^{8}$ The apparently stronger interaction of the 68-412 construct, lacking the $\mathrm{N}$-terminal basic region, with STAT3 $\beta$ protein is surprising, but it may reflect a conformation dependency for interaction (Figure 1A). Mutants with extended N-terminal deletions, which included the HLH domain (construct 123-412) and two $\alpha$-helices (constructs 184-412 and 259412) failed to bind to STAT3 $\beta$ (Figure 1A, lines 4-6). Interestingly, the mutant with the most extended deletion, that comprised the bHLH domain and all three $\alpha$-helices (construct 293412), partially restored interaction with STA3 $\beta$ (Figure 1A, line 7), thus implicating the C-terminal domain in the STRA13-STAT3 interaction. The 1-299 construct, lacking the C-terminal domain, did not show any binding activity (Figure 1A, line 8). In summary, these experiments clearly demonstrate that the helix-loop-helix region (65-108 residues) and the C-terminal part (300-412 residues) of STRA13 are essential for STAT3-binding.

\section{STRA13 binds to the tyrosine-phosphorylated forms of STAT3 $\beta$ and STAT3 $\alpha$ proteins}

The phoshorylation status of the STAT3 molecule in the cell reflects its transcriptional activity. To find which of the STAT3 isoforms preferentially binds to STRA13 protein, we treated cells with sodium pervanadate (PV), a commonly used protein-tyrosine phosphatase inhibitor. ${ }^{33}$ A 15 minute pervanadate treatment of $293 \mathrm{~T}$ cells transfected with STRA13-FLAG and STAT3-HA constructs dramatically increased the pool of tyrosinephosphorylated proteins. Co-immunoprecipitation revealed that inhibition of the tyrosine phosphatases boosted the amount of STAT3 $\beta$ protein that binds to the STRA13 protein, as compared to that in the untreated cells (Figure 1B). Re-probing of the same membrane with the antibodies specific to phospho-tyrosine showed that most of the co-immunoprecipitated STAT3 $\beta$ protein had phosphorylated tyrosine (Figure 1B).

The activation of STAT3 requires tyrosine phosphorylation at residue 705 . To confirm that STRA13 binds mostly to phosphorylated STAT3, we used STAT3 $\alpha$ mutated on tyrosine 705 . Co-expression of STRA13-FLAG and Y705-STAT3 in 293T cells with subsequent co-immunoprecipitation revealed that mutation of tyrosine 705 practically abrogates binding of STAT3 to STRA13 (Figure 1C).

Overall, this study clearly demonstrates that STRA13 interacts with both the STAT3 $\alpha$ and $\beta$ isoforms and shows a strong binding preference for transcriptionaly active (phosphorylated) proteins.

\section{STRA13 activates transcription from STAT- responsive elements GAS, ISRE and STAT3}

Preferential binding of STRA13 to activated forms of STAT3 implicates the STAT3/STRA13 complex in transcription regulation. In the search for transcription sites common to STAT3 and STRA13, we studied the ability of the STRA13 to regulate STAT-dependent cis-elements. For this, we employed the Mercury JAK-STAT Pathway Profiling system (Clontech), which includes the enhancer elements GAS (interferon- $\gamma$ activating sequence), ISRE (IFN-stimulated responsive element) and STAT3 (a modified GAS sequence), each introduced as three tandem copies upstream of the TA promoter in a luciferase reporter vector (Figure 2A). We showed that co-transfection of 293T cells with the STRA13-FLAG expression construct and a reporter construct containing the GAS, ISRE, or STAT3 elements resulted in a 25-fold, 40-fold, and fivefold activation of the basal reporter gene expression, respectively (Figure 2B), in a STRA13 dosage-dependent manner. Saturation was achieved at $\sim 0.8 \mu \mathrm{g}$ per well in six-well plates (Figure 2C). No cytokine stimulation was required to boost the STRA13-dependent transcription activation from STAT-responsive elements. As a positive control, we used luciferase reporter vectors with a $c-m y c$ or an E2F-responsive cis-element inserted upstream of the TA promoter (Clontech, Mercury Cell Cycle Profiling System). As a result, strong suppression of luciferase activity (from tenfold to 50-fold) by STRA13 overexpression was reported in the same 293T cells 
A.

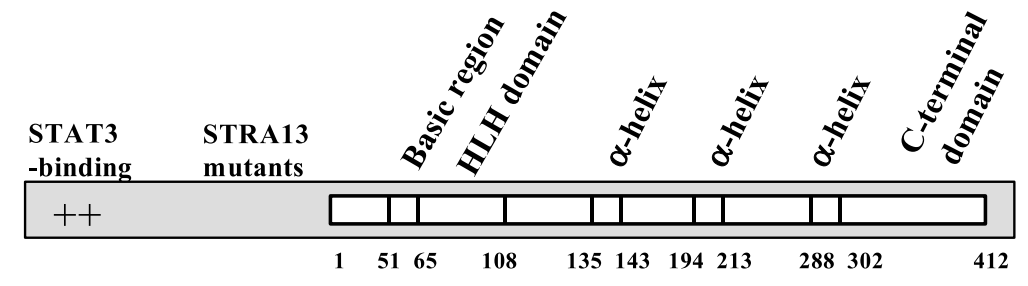

\begin{tabular}{|ccc|}
\hline+++ & $68-412$ a.a. & $\square$ \\
\hline- & $123-412$ a.a. & \\
\hline
\end{tabular}

- 184-412 a.a.

- 259-412 a.a.

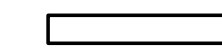

++ 293-412

- 1-299 a.a.

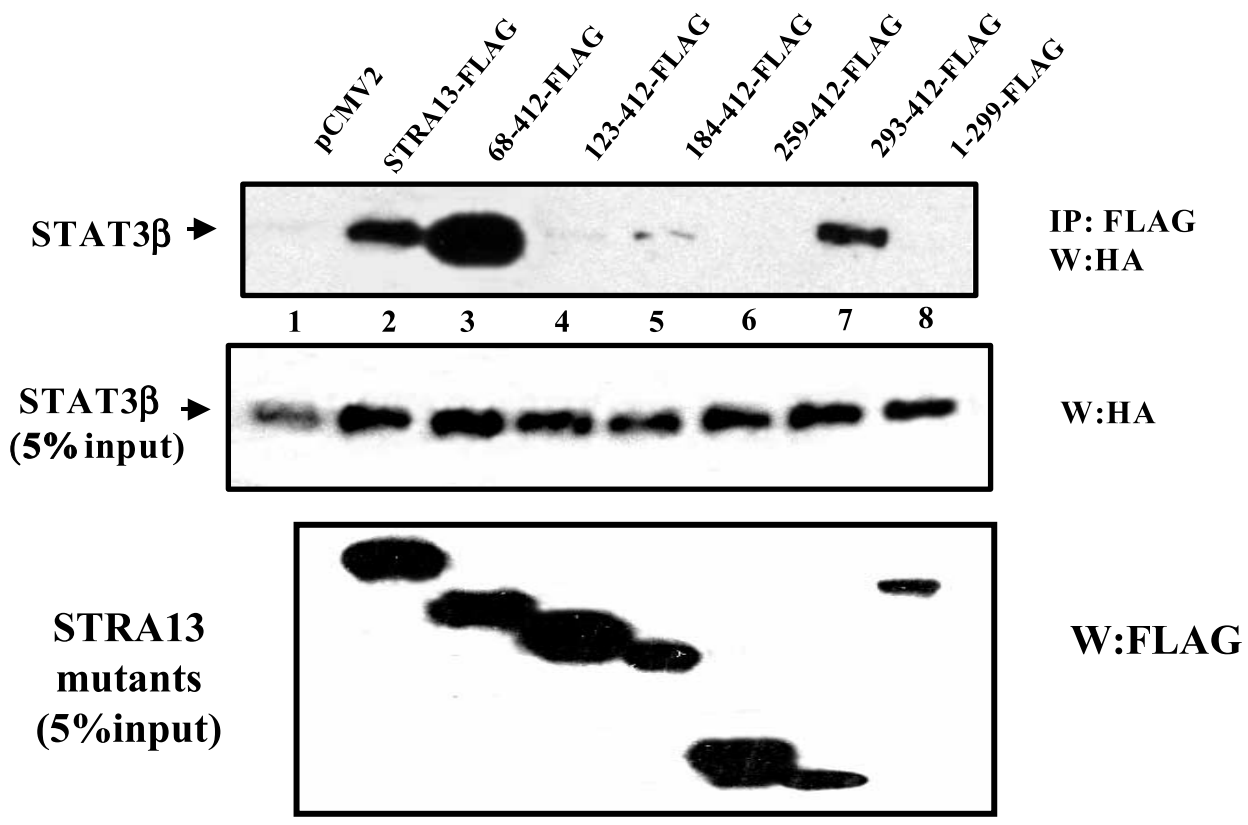

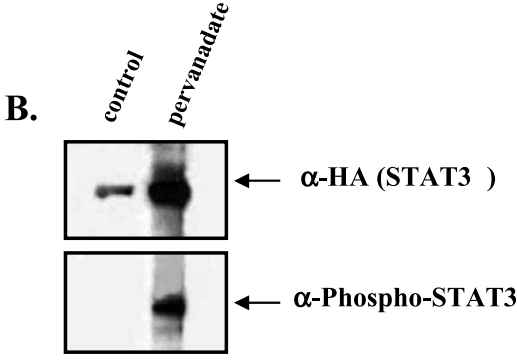

IP: $\alpha$-FLAG (STRA13)

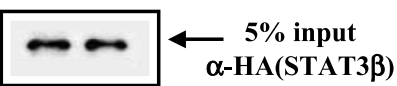

C.

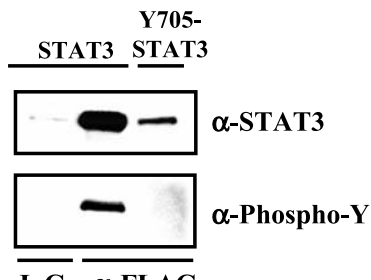

IP: $\overline{\text { IgG }} \overline{\alpha-F L A G}$

$\varpi \curvearrowleft \curvearrowleft \begin{aligned} & 10 \% \text { input } \\ & \alpha-\text { STAT3) }\end{aligned}$

Figure 1. STRA13 interacts with the STAT3 $\beta$ protein in vivo. A, Identification of the STAT3 $\beta$ binding sites within the STRA13 molecule. Indicated portions of STRA13 were fused to FLAG-tag in the pCMV2 vector and co-transfected with the STAT3 $\beta$-HA construct into 293T cells. Immunoprecipitation with anti-FLAG antibodies and analysis of immune 
A

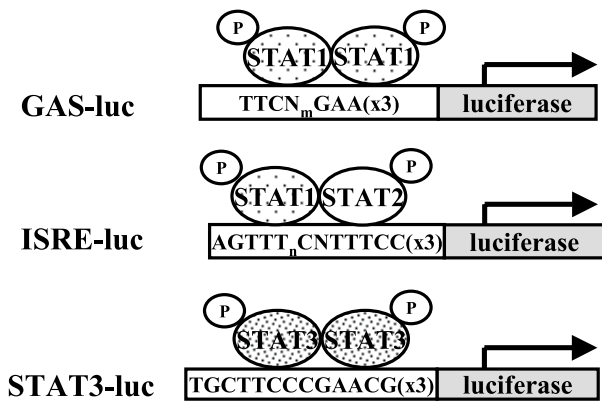

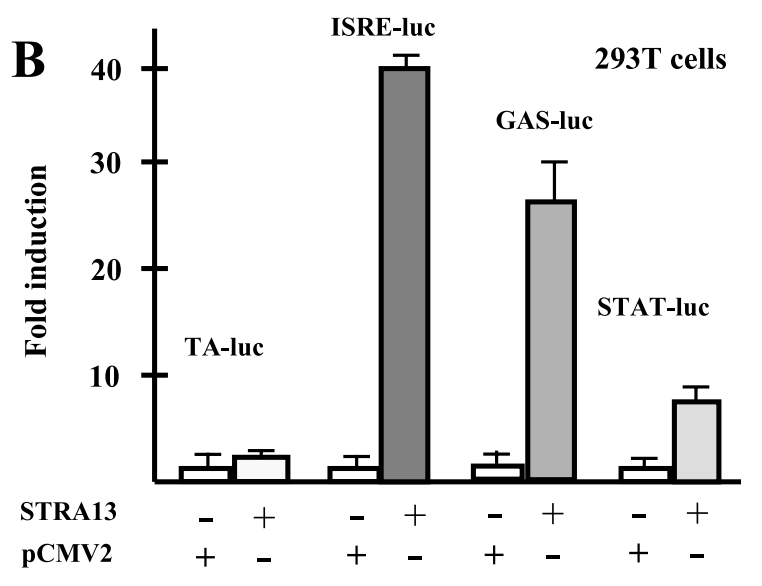

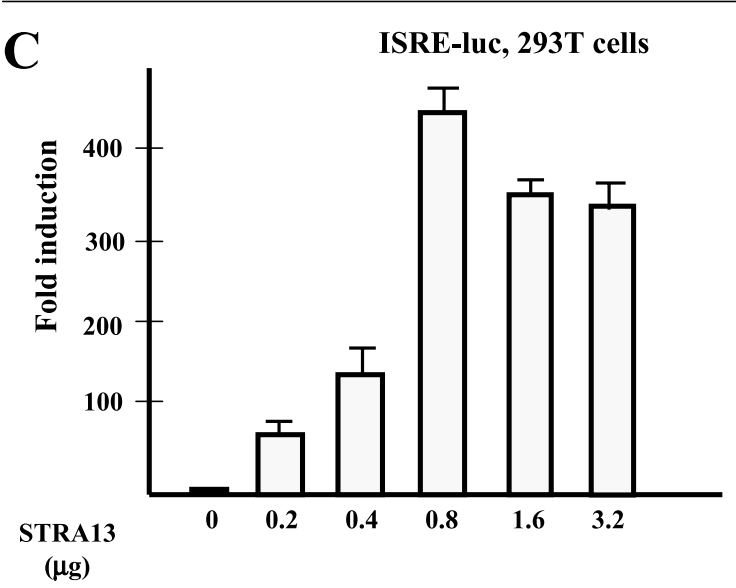

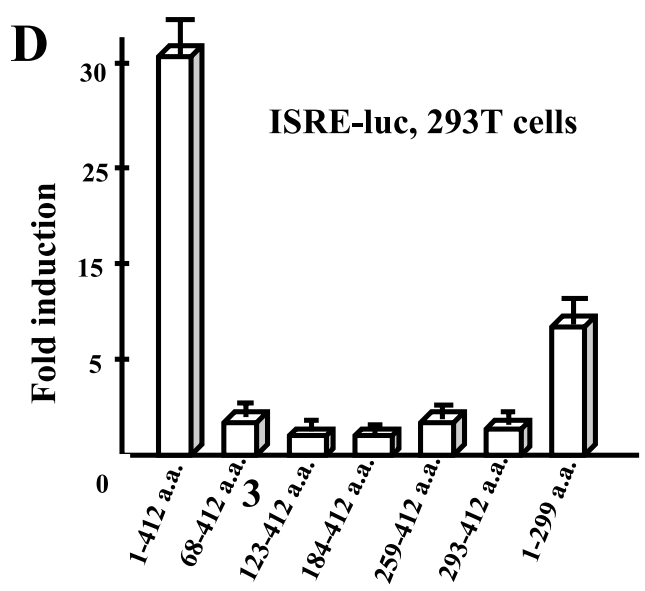

Figure 2. STRA13 stimulates transcription from STAT-dependent cis-elements. A, Schematic illustration of the luciferase reporter gene driven by STAT-dependent cis-elements used in the study. Each element is known to preferentially bind indicated STAT homo- or heterodimeric complexes. B, Overexpression of STRA13 stimulates transcription from all studied STAT-dependent cis-elements. 293T cells were transiently co-transfected with the indicated luciferase plasmids and STRA13-FLAG or empty plasmids. The experiments were repeated at least five times, error bars indicate the standard deviation of mean values. C, STRA13 activates transcription from cis-elements in a STRA13 dosagedependent manner. The ISRE-luc reporter and increased concentrations of STRA13-FLAG plasmid were co-transfected into 293 T cells. The pCMV2-FLAG vector was used as a control. D, Analysis of the transcription activation domain in STRA13. Various deletion mutants of STRA13 were assessed for their ability to activate the ISRE cis-element by luciferase assay as described above with the ISRE-luc and STRA13 mutants introduced into 293T cells. The experiments were repeated three times; error bars indicate the standard deviations. STRA13 mutants are designated according to the amino acid residues positions of their $\mathrm{N}$ and $\mathrm{C}$ termini. Fold induction was calculated in comparison with the basal luciferase activity obtained with the pCMV2 vector.

(data not shown). Luciferase vector with no insertion upstream of the TA promoter served as a negative control and showed no change in luciferase activity upon STRA13 overexpression
(Figure 2B). These data provide direct evidence that STRA13 may serve as a powerful transactivator of specifically STAT-dependent ciselements.

complexes by Western blotting using HA-antibodies were performed as described in Materials and Methods. Truncated proteins that retained the ability to interact with STAT3 $\beta$ are boxed. Filled rectangles on the schematic diagram of the STRA13 protein represent the regions important for binding to STAT3 $\beta$. B, STRA13 protein preferentially binds the phosphorylated isoform of the STAT3 $\beta$ protein. 293T cells were co-transfected with the STRA13FLAG and the STAT3 $\beta$-HA overexpression plasmids. Twenty-four hours post-transfection cells were split into two plates and on the next day one plate was treated with sodium pervanadate for 15 minutes while the other was used as a control. The immunoprecipitation (IP) procedure was performed as described above. Anti-FLAG antibodies were used for IP, antibodies indicated on the sides of each panel were used for Western analysis. C, Y705 mutation abrogates binding of STRA13 to STAT3 $\alpha$. 293T cells were transfected with the STRA13-FLAG plasmid and with STAT3 $\alpha$ or Y705/STAT3 $\alpha$ plasmids. IP and followed Western blot were performed as described above. IP with mouse IgG was used as a control. W, Western blot; $\alpha$-FLAG, anti-FLAG Abs; $\alpha$-HA, anti-HA Abs, $\alpha$-phospho-STAT3, antiphospho-STAT3 Abs; $\alpha$-STAT3 $\alpha$ (H-190), anti-STAT3 antibodies, $\alpha$-Phospho-Y, anti-phosphotyrosine antibodies. 


\section{The basic domain and the C-terminal region of STRA13 are responsible for transcription activation from cytokine-responsive elements}

Since the ISRE element in our system was the most sensitive to STRA13 overexpression, we used the ISRE-luciferase assay for mapping of the STRA13 regions responsible for transcriptional activation. 293T cells were transiently co-transfected with ISRE-luc plasmid and with different STRA13 deletion constructs (Figure 2D). In this experiment, ALLnL treatment for 24 hours prior to the lysates preparation was also used to prevent degradation of the STRA13 mutant (see above). We found that truncation of the N-terminal DNAbinding basic region (construct 68-412), as well as the extension of the $\mathrm{N}$-terminal deletion (constructs 123-412, 184-412, 259-412, 293-412), abrogated the ability of STRA13 to activate the reporter gene (Figure 3D). Truncation of the C-terminal 112 amino acid residues (construct 1-299) drastically decreased the STRA13 transcriptional activity in the ISRE-luc assay (sevenfold luciferase induction), compared to the effect of the full-length STRA13 (30-fold luciferase induction). These data illuminate the critical importance of the $\mathrm{N}$-terminal basic domain for STRA13 transactivation function. Transactivation ability of the C-terminal domain, which shows no similarity to any known protein motif, requires further investigation.

\section{STRA13 expression is induced by cytokines}

STRA13's ability to activate cytokine-responsive elements has raised the question of cytokine's possible involvement in the regulation of STRA13 transcription. It was demonstrated that promoters of certain transcription factors (c-myc, nmi, pim1, etc.) are activated with cytokines through the JAKSTAT pathway ${ }^{34-36}$ while the protein product of the cytokine-activated pim1 gene was shown to bind STAT proteins. ${ }^{37}$ Context analysis of the STRA13 promoter revealed putative binding sites for cytokine-inducible STATs and c-Myc (data not shown). Altogether these facts prompted us to assess cytokine involvement in the regulation of STRA13 transcription. In our search for a model cell line that could be used in this study, we found that the natural killer IL-2-dependent cell line
NK92 is responsive to multiple cytokines as determined by expression of IFN- $\gamma$ (data not shown). In our experimental assay NK92 cells were treated with recombinant IL-1 $\beta$, IL-2, IL-4, IL-6, IL-7, IL-12, IL-15 IL-18, IL-21, and TNF-alpha and IFN-beta. After three hours of incubation, the level of STRA13 mRNA was assessed by Northern analysis following densitometry analysis. As shown in Figure 3, IL-2, IL-6, IL-12, IL-15, TNF-alpha, and IFN-beta significantly enhanced STRA13 mRNA expression while IL-1beta, IL-4, IL-7, IL-18, and IL-21 effects were less pronounced, if visible at all, after three hours of stimulation with the indicated cytokines. Thus, this experiment clearly demonstrates contribution of at least IL-2, IL-6, IL-12, IL-15, TNF-alpha, and IFN-beta to the regulation of STRA13 mRNA expression corroborating our data on STRA13 involvement in STATdependent pathways.

\section{Roles of STRA13 and STAT3 in the regulation of Fas transcription}

The cell surface receptor Fas and its cognate ligand FasL play essential roles in apoptosis regulation and are required for immune system homeostasis. In cooperation with c-Jun, STAT3 downregulates the transcription of the Fas receptor promoter in melanoma cells contributing to the resistance of these cells to apoptosis. ${ }^{38}$ Targeted disruption of Stra13 showed that this gene product is also directly or indirectly involved in Fas-FasL mediated apoptosis, ${ }^{15}$ apparently by regulating the FasL level. The role of STRA13 in Fas receptor regulation, however, remained unclear. To determine possible involvement of STRA13 in the regulation of Fas receptor as a known STAT3 transcriptional target, we employed Fas-Luc reporter constructs and the human Jurkat cell line. Our choice of Jurkat cells, which have an activated T-cell phenotype, as a model cell line is based on the fact that Stra13 was shown to play a crucial role in the Fas-mediated apoptosis of activated $\mathrm{T}$ and B cells. ${ }^{15}$ Forced expression of STRA13 led to a three- to fivefold increase in Fas promoter-driven reporter activity in a STRA13-dosage-dependent manner (Figure 4). STAT3 $\beta$ overexpression also resulted in promoter activation, while co-expression of STRA13 and STAT3 $\beta$ led to

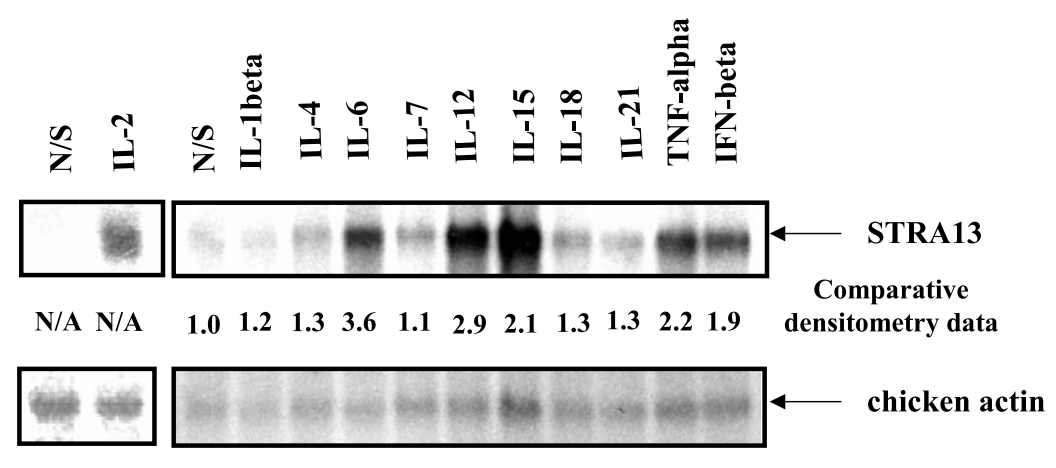

Figure 3. The endogenous STRA13 mRNA level is increased after treatment with various cytokines. Responsiveness of STRA13 expression to cytokines was studied in IL-2-dependent NK92 cells. After three hours of incubation with the indicated cytokines the total RNA was extracted and the level of STRA13 mRNA was assessed by Northern analysis. The actin mRNA was used as a loading control. 
1.5-fold suppression of the Fas-luc basal transcription (Figure 4). Under the same conditions, the expression of STAT3 $\alpha$ repressed Fas-dependent-Luc activities but failed to repress STRA13dependent activation of the Fas promoter to the same extent as STAT3 $\beta$ (Figure 4). These results imply that the STRA13/STAT3 $\beta$ and STRA13/ STAT $3 \alpha$ protein complexes have different biological properties. In line with the data on melanoma cells, ${ }^{38}$ IL-6 treatment did increase, but not significantly, the activation of the Fas promoter by the studied proteins (data not shown).

Truncation of the N-terminal basic domain (construct 68-412) or $\mathrm{COOH}$-terminal domain of STRA13 (construct 1-299) led to complete abrogation of the STRA13 activating function (data not shown). These data are in agreement with our previous findings of the critical roles of these domains in the STRA13 transactivation function (Figure 2D).

\section{STRA13 over-expression causes apoptosis}

Since Fas expression directly regulates Fas/FasLmediated apoptosis, our next step was to investigate whether cells over-expressing STRA13 undergo apoptosis. To assess the involvement of human STRA13 in regulation of apoptosis via transient expression, we used 293T cells, distinguished with a high tranfection rate.

We applied the TUNEL assay to estimate the proportion of $293 \mathrm{~T}$ cells that undergo apoptosis during transient expression of the mock pCMV2-

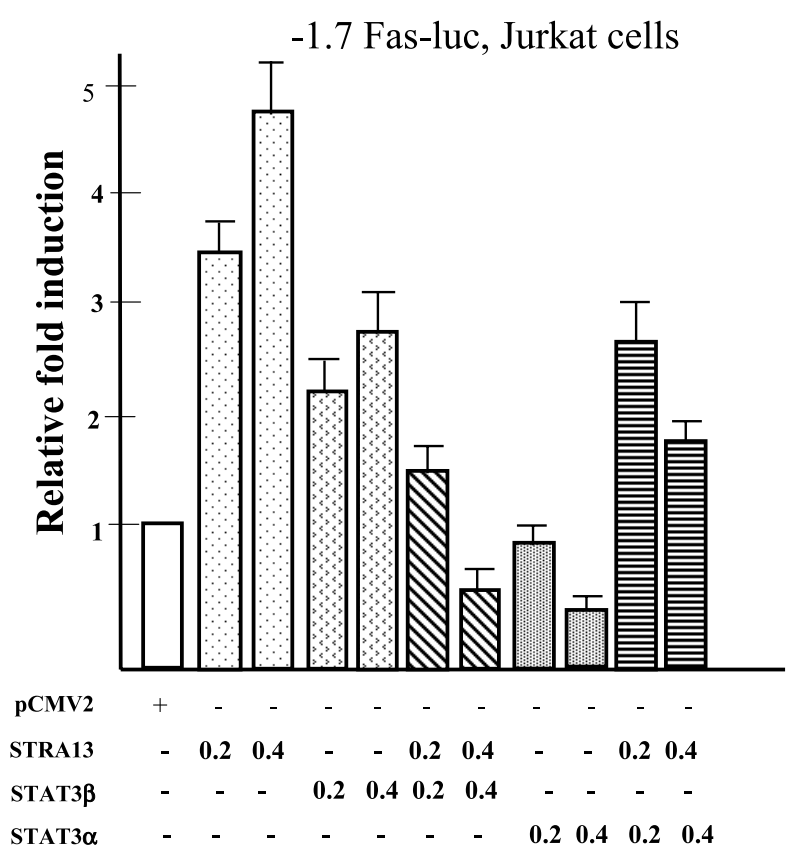

Figure 4. Co-expression of STRA13 with STAT3 $\alpha$ or STAT3 $\beta$ produces different effects on STRA13-dependent activation of the $-1.7 \mathrm{~kb}$ Fas-promoter. Jurkat cells were transfected with the $-1.7 \mathrm{~kb}$ Fas-luc reporter construct and the indicated amounts of the STRA13, STAT $3 \alpha$ or STAT3 $\beta$ plasmids. The results shown are representative of five independent experiments.
FLAG vector, the STRA13-FLAG, and the 259412-FLAG constructs. Apoptotic cells were counted three days after transient transfection. Cells transfected with STRA13-FLAG showed a fourfold increase in apoptotic cells (Figure 5A) as compared to the mock vector, whereas transfection with the C-terminal part of STRA13 protein did not change the amount of apoptotic cells, compared to the control. The extent of the STRA13 effect on apoptosis in this assay is, most likely, underestimated because of the limited efficiency of transient transfection $(\sim 60 \%)$. Immunostaining demonstrated that many of the cells expressing STRA13/FLAG are TUNEL-positive and have apoptotic dense nuclei. An apoptotic phenotype was associated with altered localization of STRA13, which resulted in its dispersed dotted distribution in cytoplasm (Figure 5B). To confirm the involvement of STRA13 in Fas-dependent apoptosis by regulation of the endogenous Fas gene expression, quantitative RT-PCR analysis was applied to the same set of transfected cells. We showed that STRA13 overexpression resulted in the increase of the endogenous Fas expression in 293 cells when compared to experiments with empty vector or with the STRA13 construct lacking the bHLH domain (Figure 5C). To investigate the effect of STRA13 and STAT3 $\beta$ co-expression on the apoptotic process, we compared the amount of apoptotic cells in 293T cell line transfected with STRA13 and STAT3 $\beta$ constructs separately or simultaneously. Transient expression of either STRA13 or STAT3 $\beta$ during the following 48 hours resulted in an increase in apoptotic cells compared to the mock transfection, while co-expression of these two proteins resulted in the decrease of apoptotic cells, compared to the effects of STRA13 or STAT3 $\beta$ alone (Figure 5D). Co-expression of STAT3 $\beta$ with the N-truncated STRA13 version did not lead to any significant change in the amount of apoptotic cells, compared to STAT3 $\beta$ alone. These data are in line with our observation that STRA13 and STAT3 $\beta$ co-expression decreases luciferase transcription from the Fas-luc promoter, while each of these proteins alone has a transactivating effect. These data, overall, provide strong support towards our hypothesis that STRA13, along with STAT3, functions as a regulator of the Fas-dependent apoptotic pathway.

\section{Discussion}

Our study provides biochemical and functional evidence of association between the bHLH transcription factor STRA13 and the transcriptional regulator STAT3. We demonstrate that binding of STRA13 to STAT3 $\beta$ takes place in the mammalian cell and recruits the helix-loop-helix and the C-terminal domains of STRA13. While the HLH domain is evolutionarily conserved and is known to serve in the protein-protein interaction function, involvement of the C-terminal domain is 

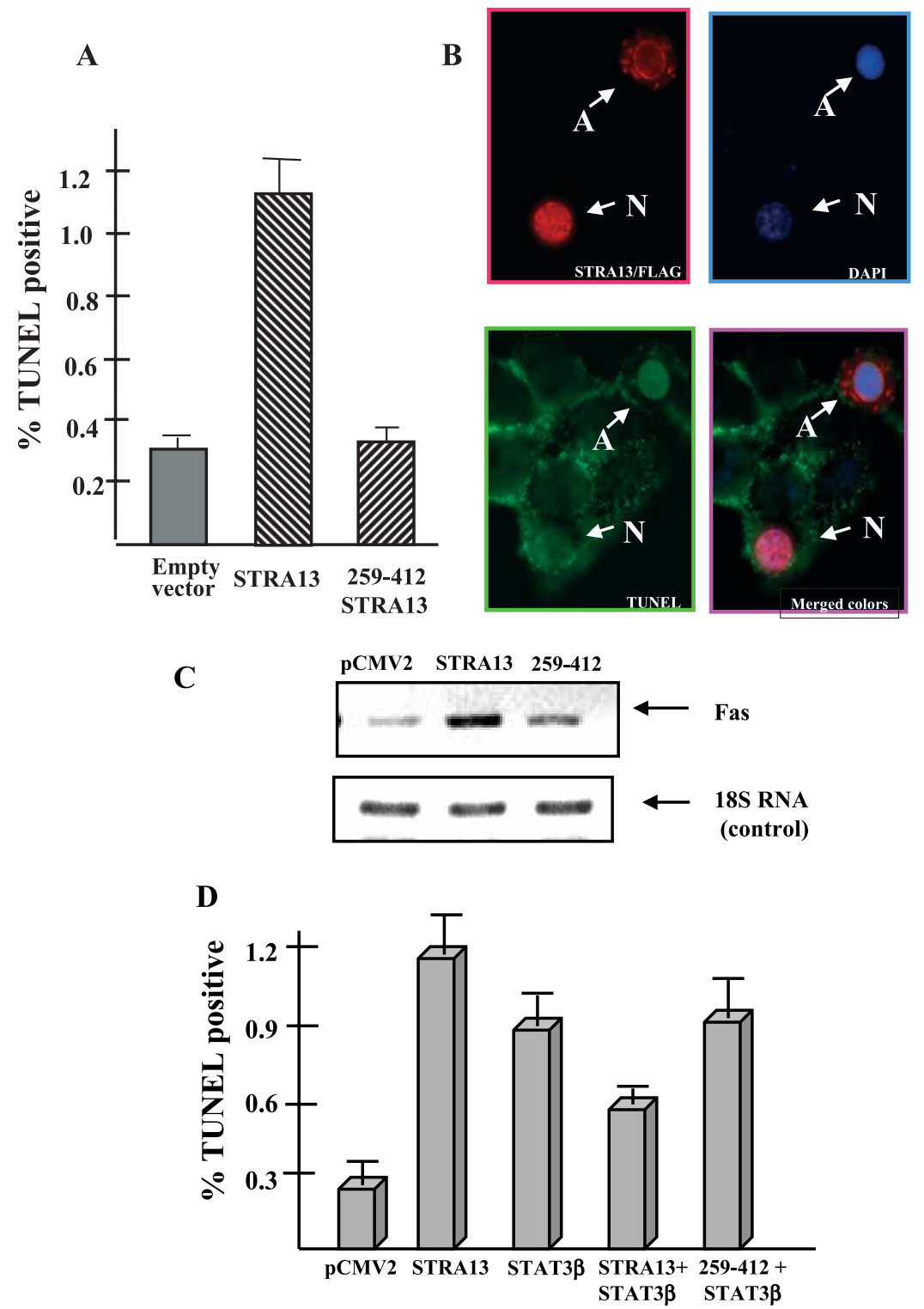

Figure 5. STRA13 overexpression induces cell death in $293 \mathrm{~T}$ cells. A， STRA13-FLAG，259-412-FLAG (N-truncated STRA13) plasmids or empty pCMV2 vector were transfected in 293T cells. At 48 hours post-incubation, cells were cultured on coverslips. Cell death was assayed by the TUNEL method. The means of three independent experiments are shown. B, Immunostaining of TUNELassayed $293 \mathrm{~T}$ cells from the experiment described in A with an antiFLAG M2 Abs (red) showing STRA13/FLAG positive cells. DAPI was used to counterstain the nuclei. The arrows indicate apoptotic (A) and normal (N) STRA13-positive cells. C, Quantitative RT-PCR performed on RNA isolated from the cell lines transiently transfected with STRA13 construct or empty vector or STRA13 mutant construct as controls. $18 \mathrm{~S}$ rRNA expression was used as an internal standard. $D$, The effect of STRA13 and STAT3 $\beta$ co-expression on the apoptotic process in $293 \mathrm{~T}$ cells transiently transfected with the indicated plasmids. Cell death was assayed by the TUNEL method at 48 hours post-incubation. The means of three independent experiments are shown. especially interesting because no evolutionary conserved regions have been recognized in this area. It is possible that the C-terminal domain is responsible for the specificity of STRA13 interaction with STAT3. Two STRA13 homologs have been described recently, (SHARP-1 $1^{39,40}$ and DEC2 ${ }^{41}$ ), with their HLH domains almost identical (97$100 \%$ ) with that of STRA13 and their C-terminal regions highly diverged. It would be interesting to find out if these proteins can interact with the STATs, and, if so, to evaluate the possible role of the $\mathrm{C}$ terminus in the specificity of such interactions. It has been shown that transcriptional coactivators, such as CBP, p300, MCM5, and NcoA/ SRC $1 \mathrm{a}^{42-45}$ bind to STATs via their C-terminal transactivation domain (TAD). Here, we report that STRA13 binds to the STAT3 isoform $\beta$ that lacks the C-terminal TAD. The fact that STAT3 $\beta$ and STRA13 were able to interact without the TAD region suggested a repressing rather than activating potential for the STRA13/STAT3 complexes, similar to the described STAT5/SMRT complex that is formed through the STAT5 N-terminal coiled-coil region. ${ }^{46}$

Taking into consideration our finding that the transcriptional regulator STRA13 exhibited a higher affinity to the transcriptionally active, or phosphorylated, isoforms of STAT3, we searched for the common transcriptional targets that can be regulated with the STRA13/STAT3(PY) complex. First, we investigated STRA13 activity on the known STAT-dependent cis-elements. These elements can be classified into two groups. The prototype of the first one is the interferon-stimulated response element ISRE, ${ }^{47}$ which STAT1/ STAT2 heterodimers were shown to bind. STAT1, STAT2, STAT3, STAT4, STAT5a, STAT5b, and STAT6 protein complexes were shown to bind another class of cis-elements, known as GAS motifs. ${ }^{48}$ The STAT3 element, a variant of the GAS motif that preferentially interacts with STAT3, was also used in the current study. 
STRA13-dependent transactivation, shown on the ISRE, GAS, and STAT cis-elements in 293T cells, demonstrated STRA13 ability to induce transcription. This transactivation was shown only for the STAT-dependent cis-elements, while STRA13 suppressed, as expected, transcription from other cis-elements used in the study (c-myc-dependent, E2F-dependent elements). The ability of STRA13 to activate STAT-dependent promoters without cytokine stimulation may be explained by a selfsufficiency of this nuclear protein for transactivation once it is transcriptionally up-regulated. The basic STRA13 domain, generally considered to possess a DNA-binding function, ${ }^{49}$ was found in our study to be primarily responsible for the transactivation from STAT-responsive elements. Thus, it may be considered as the additional evidence that STRA13 is able to activate the promoter directly through DNA binding. The C-terminal part of the protein, although dispensable, was also shown to play a role in the transactivation process. Until recently, Stra13 was considered to be a transcriptional repressor. $^{9-11}$ Targeted disruption of Stra13, however, presented indirect evidence of its involvement in transcription activation of certain genes. For example, Stra13 $3^{-1-}$, in comparison with wild-type $\mathrm{CD}^{+} \mathrm{T}$ cells, produced less IFN- $\gamma$ and IL-4. Reintroduction of Stra13 in these cells fully rescued IFN- $\gamma$ and IL-4 expression in STRA13 ${ }^{-1-}$ cells upon their differentiation into Th1 cells, indicating that expression activation of these cytokines depends on the Stra13 function. ${ }^{15}$ In line with these observations, our experiments on STATdependent cis-elements produced the first direct evidence of STRA13 potent transactivation properties.

Binding of STRA13 to cytokine-regulated ciselements prompted us to examine the effect of cytokines on the STRA13 mRNA expression. It was reported that transcription factors $c-m y c, n m i$ and pim-1 are transcriptionally activated upon cytokine stimulation through the JAK-STAT pathway, ${ }^{35,50}$ while the product of one of these genes, pim-1 was shown to bind STAT proteins. ${ }^{37}$ Therefore, the fact that the STRA13 promoter contains putative binding sites for cytokine-inducible STATs, c-Myc and NF-kappaB, was another indirect point of evidence of cytokine involvement in STRA13 transcriptional regulation. We demonstrated that endogenous STRA13 expression was significantly increased upon treatment with IL-2, IL-6, IL-12, IL-15, TFN-alpha, and IFN-beta. Such a broad spectrum of cytokines affecting STRA13 transcription implies an important role(s) for STRA13 in functionally pleiotropic processes of cell growth, differentiation, apoptosis, immune response, etc. regulated by STATs. It is known, for instance, that IL-2 and IL-15 activate STAT5, IL-6 is involved in STAT3 activation, IL-12 is responsible for STAT4 phosphorylation, and IFN-beta activates the STAT1 transcription factor. ${ }^{51,52}$ It was also found recently that TNF-alpha, along with its known role in NF-kappaB activation, induces the activation of the tyrosine kinases Jak1 and Tyk2 in human B cells subsequently activating STAT3 and STAT5 $\beta$ proteins. ${ }^{53}$ Taken together with the data on STRA13-dependent activation of a variety of STAT-specific cis-elements, these observations emphasize STRA13 importance as an integral part of the STAT-signaling pathways.

Our next step was to pursue the functional significance of STRA13 interaction with STAT3 by studying their activity on the STAT3-specific Fas promoter. In several studies oncogenic function of STAT3 signaling was associated with antiapoptosis. ${ }^{54-56}$ V.I. et al. have recently shown that STAT3 anti-apoptotic activity could be mediated through suppression of Fas expression in melanoma cell lines. ${ }^{38}$ The pro-apoptotic role of the Stra13 gene was recently proposed based on an experiment on targeted gene disruption. Mouse Stra13 ${ }^{-/-} \mathrm{CD}^{+} \mathrm{T}$ cells failed to appropriately upregulate FasL expression, leading to resistance of activated T and B cells to the Fas-mediated apoptosis and, subsequently, to their accumulation. ${ }^{15}$ The resulting phenotype, a lupus-like systemic autoimmunity, was similar to that produced by mutations in the Fas or FasL genes, ${ }^{57,58}$ illuminating Stra13's pivotal role in the regulation of peripheral T cells apoptosis. The association of STRA13 with activated STAT3 (this study) and the implication of both proteins in the Fas-dependent apoptotic molecular pathway ${ }^{15,38}$ prompted us to elucidate the possible role of STRA13/STAT3 in this process.

In our experiments with the Fas promoter performed on Jurkat cells, STRA13 over-expression resulted in transcriptional activation of the promoter. STAT3 $\beta$ also demonstrated a significant, albeit less pronounced, activation effect. In agreement with our data, the STAT3 $\beta$ isoform as a dominantnegative of STAT3 $\alpha$ was also reported to activate transcription from the Fas promoter in melanoma cell lines. ${ }^{38}$ STAT $3 \alpha$, in our experiment, suppressed the Fas-luc promoter, which was also in line with the experiments on melanoma cells. ${ }^{38}$ STAT3 $\alpha$ and $\beta$ possess different biochemical properties and have non-overlapping biological functions. STAT3 $\beta$, a constitutively activated isoform of STAT3 $\alpha$ that lacks the C-terminal domain, attenuates STAT3 $\alpha$ transcriptional activities. ${ }^{23,59}$

Unexpectedly, co-expression of both STRA13 and STAT3 $\beta$ led to suppression of the transcription. Co-expression of STRA13 and STAT3 $\alpha$, on the other hand, alleviated the STAT3 $\alpha$-dependent suppression (Figure 4). These data suggest that the balance between STRA13 on one side and STAT $3 \alpha /$ STAT3 $\beta$ on the other plays an important role in the regulation of the Fas promoter and, thus, may contribute to the cell survival decision.

Impact of STRA13 expression on apoptosis was than tested in a cell culture functional assay. Using the TUNEL assay, we showed that STRA13 transient expression caused a $\sim$ fourfold increase in apoptotic cells, clearly implicating STRA13 in apoptosis induction. We also obtained direct confirmation of the regulation of Fas expression by 
STRA13 from the quantitative RT-PCR analysis of endogenous Fas expression. STRA13 overexpression increased the amount of Fas mRNA compared to overexpression of the empty vector.

The paradoxical effect of STRA13 and STAT3 $\beta$ co-expression on the Fas-luc promoter found its support in our functional apoptosis assay leading to decreases in apoptotic cells number when compared to the effects of the proteins expressed alone (Figure 5D). Further experiments are required for deciphering the molecular mechanisms of this effect.

In conclusion, we characterize STRA13 and STAT3 as protein partners involved in complex regulation of STAT transcriptional targets. Our study provides a consistent line of evidence that the bHLH transcription factor STRA13 may serve as a transcriptional activator as an integral part of the STAT transcriptional network. Through this network STRA13 may contribute to the regulation of critical processes of cell survival and growth, known to be disregulated in cancer cells. Under the most simplistic scenario that may be proposed, STRA13 mRNA overexpression caused by pVHL deficiency, hypoxia or other stimuli may have either an apoptotic or anti-apoptotic effect, depending on the availability of activated STAT isoforms. Indeed, many cancer cells are known to express high levels of STAT3 $\beta$, which may interact with STRA13 and suppress apoptosis via Fasdependent mechanism. STATs, however, are also implicated in regulation of growth arrest through p19 and p21 and G1 to S cell cycle progression through Pim or c-Myc. ${ }^{18}$ Thus, more investigation into the STAT network is required to elucidate all possible consequences of STRA13/STAT3 interaction.

\section{Materials and Methods}

\section{Cell lines and reagents}

The human $293 \mathrm{~T}$ embryonic kidney cell line, the mouse fibroblasts NIH3T3 cell line and the T-lymphoma Jurkat-E6 cell line were obtained from the American Type Culture Collection (Rockville, MD) and cultured in media recommended by the supplier. The human NK-92 natural killer cell line was maintained in RPMI 1640 medium containing $10 \%$ (v/v) fetal calf serum, $2 \mathrm{mM}$ L-glutamine, 100 i.u. of penicillin, $100 \mu \mathrm{g}$ of streptomycin, and 200 i.u. of recombinant human IL-2. Before cytokine stimulation experiments, cells were maintained at a density of $10^{6} \mathrm{ml}^{-1}$ in medium lacking IL-2 for 14 hours. IL-2 (100 i.u./ml), IL-12 (100 i.u./ml), and IL-18 $(100 \mathrm{ng} / \mathrm{ml})$ were used for stimulation. The monoclonal anti-FLAG M2 antibody was purchased from Sigma. The rat monoclonal HA (clone 3F10) antibody was purchased from Roche Molecular Biochemicals, rabbit polyclonal phosphotyrosine antibodies were purchased from Cell Signaling Technology, rabbit polyclonal STAT3 (H-190) antibodies were purchased from Santa Cruz Biotechnology, Inc. Secondary anti-mouse and anti-rabbit IgG for Western blots were purchased from Santa Cruz Biotechnology, secondary Alexa Fluor 488 anti-mouse IgG for immunostaining of cells were purchased from Molecular Probes, Inc.

\section{Plasmid constructs}

STRA13 and a series of its deletion mutants were generated by amplification of the fragments from the human cDNA and cloned into the EcoRI-XbaI sites of pCMV2FLAG vector. The constructs have been verified by sequencing. STAT3 $\beta$-pCMV-HA plasmid for STRA13 interactors was generated by subcloning of the $3.2 \mathrm{~kb}$ EcoRI-XhoI insertion from the pACT2 plasmid obtained by screening of the fetal kidney cDNA Matchmaker cDNA library (Clontech) for STRA13 interactors. The luciferase reporter plasmids with the cytokine-responding cis-acting enchancer elements inserted before TATAbox were purchased from Clontech. The luciferase reporter driven by $1.7 \mathrm{~kb}$ or $460 \mathrm{bp}$ of the human Fas promoter were kindly provided by $\mathrm{Dr}$ L. B. OwenSchaub (Division of Biomedical Sciences, University of California, Riverside, CA). Stat $3 \alpha$ inserted into pRcCMV plasmid was obtained from Dr J. Bromberg (Rockefeller University and Memorial Sloan Kettering Cancer Center, New York, NJ).

\section{Immunostaining and TUNEL assay}

293T cells were transiently transfected with STRA13/ pCMV2, 259-412/pCMV2 (N-truncated STRA13 construct) or an empty pCMV2 vector using Lipofectamin $^{\mathrm{TM}} 2000$ (Gibco BRL). Seventy-two hours posttransfection cells were cultured on coverslips overnight. In the morning cells were washed in PBS, fixed in $4-7 \%$ $(\mathrm{w} / \mathrm{v})$ paraformaldehyde for 30 minutes and permeabilized with $0.1 \%(\mathrm{v} / \mathrm{v})$ Triton $\mathrm{X}-100$ for five minutes. After pre-incubation with $1 \%(\mathrm{v} / \mathrm{v})$ fetal bovine serum, cells were immunostained with anti-FLAG M2 mouse $\mathrm{mAb}$. Goat anti-mouse antibodies with Alexa Fluor TM 594 (red) were used to visualize the anti-FLAG mouse $\mathrm{mAb}$. For TUNEL assay, washed cells were treated according to the protocol for In Situ Cell Death Detection Kit (Roche). Fluorescein-12-dUTP-labeled DNA (green) was visualized by confocal microscopy $(60 \times)$. Cells were mounted in mounting media with DAPI for nuclei staining (Vector). At least 2000 cells from ten different fields were examined in each experiment, and the cell death was expressed as a percentage of TUNEL-positive cells.

\section{Northern blot analysis}

The Trizol ${ }^{\mathrm{TM}}$ procedure (Life Technologies) was employed for isolation of total RNA from cells. Electrophoresis in formaldehyde gels and Northern blot analysis were performed as described. ${ }^{6}$ Quantification of Northern hybridization signals was done with the Cyclone Storage Phosphor System (Packard) and the manufacturer's software package.

\section{Relative quantitative RT-PCR analysis}

Total RNA from 293T cells was isolated using RNeasy Mini Kit (Quagen). SuperScript One-step RT-PCR Kit (Invitrogen) was employed for RT-PCR reactions. The $18 \mathrm{~S}$ rRNA Primers and Competimers (Ambion) were used as for quantitative analysis of gene expression. 


\section{Transient transfection and luciferase reporter assay}

For transient transfection 293T or T-lymphoma JurkatE6 cell lines were grown in six-well plates to $70-80 \%$ confluence and co-transfected with the indicated reporter constructs and the plasmids containing STRA13 (or its derivatives) and (or) STAT3 $\alpha$ or $\beta$ using Lipofecta$\min ^{\mathrm{TM}} 2000$ reagent (Stratagene). At 40 hours post-transfection the recipient cells were divided, when needed, into two equal volumes that were either left untreated or treated for eight hours with $10 \mathrm{ng} / \mathrm{ml}$ of IL-6 (Calbiochem). After treatment luciferase activity was detected by mixing $20 \mu \mathrm{l}$ of the extract with $100 \mu \mathrm{l}$ of luciferase substrate (Promega) and measured in a MicroLumat LB96P luminometer (EG\&G Berthold). The luciferase activity was normalized based on extract protein concentration. Fold induction was calculated by dividing the treated samples by the non-treated control for each reporter construct. Each experiment was repeated five times, after that the mean and standard deviation were calculated.

\section{Co-immunoprecipitation and Western blot analysis}

293T cells were transfected using Superfect (Quagen) according to the manufacturer's recommendations. Forty eight hours after transfection the cells were lysed in RIPA buffer (150 mM NaCl, $50 \mathrm{mM}$ Hepes ( $\mathrm{pH} 7.4)$, $10 \mathrm{mM}$ NaF, $1 \mathrm{mM}$ EDTA, $2 \mathrm{mM}$ orthovanadate, $10 \%$ $(\mathrm{v} / \mathrm{v})$ glycerol, $1 \%(\mathrm{v} / \mathrm{v})$ Triton, $1 \%(\mathrm{w} / \mathrm{v})$ deoxychelate, $0.5 \%(\mathrm{w} / \mathrm{v})$ SDS, protease inhibitors), the lysates were sheared by ultrasound and cleared by centrifugation at $13,000 \mathrm{rpm}$ for 15 minutes at $4{ }^{\circ} \mathrm{C}$. Cell lysates containing equal amount of total protein were incubated for four hours at $4{ }^{\circ} \mathrm{C}$ with $50 \mu \mathrm{l}$ of $30 \%$ (w/v) protein G-Sepharose beads (Stratagene) and anti-FLAG monoclonal antibody or control (IgG) antibodies. The beads were washed extensively with lysis buffer and bound proteins were fractionated on SDS/8-16\% PAGE, transferred to a nitrocellulose membrane, and incubated with the anti-HA antibodies. Flag or HA-fused proteins were visualized using the ECL kit (DURA, Pierce).

\section{Yeast two-hybrid screen}

The entire open reading frame of STRA13 (411 residues) was inserted into EcoRI-BamHI restriction sites of the pAS2-1 expression vector (Clontech) that was used as a bait. The two-hybrid screening was performed as described. ${ }^{8}$

\section{Acknowledgements}

We thank Laura Geil for help with the manuscript editing. This publication has been funded in whole or in part with Federal funds from the National Cancer Institute, National Institute of Health, under Contracts NO1-CO-12400 and NO1CO-56000. The content of this publication does not necessarily reflect the views or policies of the department of Health and Human Services, nor does mention of trade names, commercial products, or organizations imply endorsement by the US Government.

\section{References}

1. Ivan, M. \& Kaelin, W. G., Jr (2001). The von HippelLindau tumor suppressor protein. Curr. Opin. Genet. Dev. 11, 27-34.

2. Maxwell, P. H., Wiesener, M. S., Chang, G. W., Clifford, S. C., Vaux, E. C., Cockman, M. E. et al. (1999). The tumour suppressor protein VHL targets hypoxia-inducible factors for oxygen-dependent proteolysis. Nature, 399, 271-275.

3. Gnarra, J. R., Zhou, S., Merrill, M. J., Wagner, J. R., Krumm, A., Papavassiliou, E. et al. (1996). Posttranscriptional regulation of vascular endothelial growth factor mRNA by the product of the VHL tumor suppressor gene. Proc. Natl Acad. Sci.USA, 93, 10589-10594.

4. Iliopoulos, O., Levy, A. P., Jiang, C., Kaelin, W. G., Jr \& Goldberg, M. A. (1996). Negative regulation of hypoxia-inducible genes by the von Hippel-Lindau protein. Proc. Natl Acad. Sci. USA, 93, 10595-10599.

5. Dang, C. V. \& Semenza, G. L. (1999). Oncogenic alterations of metabolism. Trends Biochem. Sci. 24, 68-72.

6. Ivanov, S. V., Kuzmin, I., Wei, M. H., Pack, S., Geil, L., Johnson, B. E. et al. (1998). Down-regulation of transmembrane carbonic anhydrases in renal cell carcinoma cell lines by wild-type von Hippel-Lindau transgenes. Proc. Natl Acad. Sci. USA, 95, 12596-12601.

7. Ivanov, S. V., Liao, S. Y., Ivanova, A. V., DanilkovitchMiagkova, A., Tarasova, N., Weirich, G. et al. (2001). Expression of hypoxia-inducible cell-surface transmembrane carbonic anhydrases in human cancer. Am. J. Pathol. 158, 905-919.

8. Ivanova, A. V., Ivanov, S. V., Danilkovitch-Miagkova, A. \& Lerman, M. I. (2001). Regulation of STRA13 by the von Hippel-Lindau tumor suppressor protein, hypoxia, and the UBC9/ubiquitin proteasome degradation pathway. J. Biol. Chem. 276, 15306-15315.

9. Boudjelal, M., Taneja, R., Matsubara, S., Bouillet, P., Dolle, P. \& Chambon, P. (1997). Overexpression of Stra13, a novel retinoic acid-inducible gene of the basic helix-loop-helix family, inhibits mesodermal and promotes neuronal differentiation of P19 cells. Genes Dev. 11, 2052-2065.

10. Sun, H. \& Taneja, R. (2000). Stra13 expression is associated with growth arrest and represses transcription through histone deacetylase (HDAC)dependent and HDAC-independent mechanisms. Proc. Natl Acad. Sci. USA, 97, 4058-4063.

11. Dhar, M. \& Taneja, R. (2001). Cross-regulatory interaction between Stra13 and USF results in functional antagonism. Oncogene, 20, 4750-4756.

12. Shen, M., Kawamoto, T., Yan, W., Nakamasu, K., Tamagami, M., Koyano, Y. et al. (1997). Molecular characterization of the novel basic helix-loop-helix protein DEC1 expressed in differentiated human embryo chondrocytes. Biochem. Biophys. Res. Commun. 236, 294-298.

13. Shen, M., Kawamoto, T., Teramoto, M., Makihira, S., Fujimoto, K., Yan, W. et al. (2001). Induction of basic helix-loop-helix protein DEC1 (BHLHB2)/Stra13/ Sharp2 in response to the cyclic adenosine monophosphate pathway. Eur. J. Cell. Biol. 80, 329-334.

14. Yun, Z., Maecker, H. L., Johnson, R. S. \& Giaccia, A. J. (2002). Inhibition of PPARgamma2 gene expression by the HIF-1-regulated gene DEC1/Stra13. A mechanism for regulation of adipogenesis by hypoxia. Dev. Cell, 2, 331-341. 
15. Sun, H., Lu, B., Li, R. Q., Flavell, R. A. \& Taneja, R. (2001). Defective $T$ cell activation and autoimmune disorder in Stra13-deficient mice. Nature Immunol. 2, 1040-1047.

16. Seimiya, M., Bahar, R., Wang, Y., Kawamura, K., Tada, Y., Okada, S. et al. (2002). Clast5/Stra13 is a negative regulator of B lymphocyte activation. Biochem. Biophys. Res. Commun. 292, 121-127.

17. Meyer, T., Begitt, A., Lodige, I., van Rossum, M. \& Vinkemeier, U. (2002). Constitutive and IFNgamma-induced nuclear import of STAT1 proceed through independent pathways. EMBO J. 21, 344-354

18. Wegenka, U. M., Buschmann, J., Lutticken, C., Heinrich, P. C. \& Horn, F. (1993). Acute-phase response factor, a nuclear factor binding to acute-phase response elements, is rapidly activated by interleukin-6 at the posttranslational level. Mol. Cell. Biol. 13, 276-288.

19. Hirano, T., Ishihara, K. \& Hibi, M. (2000). Roles of STAT3 in mediating the cell growth, differentiation and survival signals relayed through the IL-6 family of cytokine receptors. Oncogene, 19, 2548-2556.

20. Bowman, T., Garcia, R., Turkson, J. \& Jove, R. (2000). STATs in oncogenesis. Oncogene, 19, 2474-2488.

21. Turkson, J. \& Jove, R. (2000). STAT proteins: novel molecular targets for cancer drug discovery. Oncogene, 19, 6613-6626.

22. Schaefer, T. S., Sanders, L. K. \& Nathans, D. (1995) Cooperative transcriptional activity of Jun and Stat3 beta, a short form of Stat3. Proc. Natl Acad. Sci. USA, 92, 9097-9101.

23. Caldenhoven, E., van Dijk, T. B., Solari, R., Armstrong, J., Raaijmakers, J. A., Lammers, J. W. et al. (1996). STAT3beta, a splice variant of transcription factor STAT3, is a dominant negative regulator of transcription. J. Biol. Chem. 271, 13221-13227.

24. Chakraborty, A., White, S. M., Schaefer, T. S., Ball, E. D., Dyer, K. F. \& Tweardy, D. J. (1996). Granulocyte colony-stimulating factor activation of Stat3 alpha and Stat 3 beta in immature normal and leukemic human myeloid cells. Blood, 88, 2442-2449.

25. Shao, H., Quintero, A. J. \& Tweardy, D. J. (2001) Identification and characterization of cis elements in the STAT3 gene regulating STAT3 alpha and STAT3 beta messenger RNA splicing. Blood, 98, 3853-3856.

26. Schaefer, T. S., Sanders, L. K., Park, O. K. \& Nathans, D. (1997). Functional differences between Stat3alpha and Stat3beta. Mol. Cell. Biol. 17, 5307-5316.

27. Sasse, J., Hemmann, U., Schwartz, C., Schniertshauer, U., Heesel, B., Landgraf, C. et al. (1997). Mutational analysis of acute-phase response factor/Stat3 activation and dimerization. Mol. Cell. Biol. 17, 4677-4686.

28. Ni, Z., Lou, W., Leman, E. S. \& Gao, A. C. (2000) Inhibition of constitutively activated Stat 3 signaling pathway suppresses growth of prostate cancer cells. Cancer Res. 60, 1225-1228.

29. Niu, G., Heller, R., Catlett-Falcone, R., Coppola, D., Jaroszeski, M., Dalton, W. et al. (1999). Gene therapy with dominant-negative Stat3 suppresses growth of the murine melanoma B16 tumor in vivo. Cancer Res. 59, 5059-5063.

30. Burke, W. M., Jin, X., Lin, H. J., Huang, M., Liu, R., Reynolds, R. K. \& Lin, J. (2001). Inhibition of constitutively active Stat3 suppresses growth of human ovarian and breast cancer cells. Oncogene, 20, 7925-7934.

31. Xia, Z., Sait, S. N., Baer, M. R., Barcos, M., Donohue,
K. A., Lawrence, D. et al. (2001). Truncated STAT proteins are prevalent at relapse of acute myeloid leukemia. Leuk. Res. 25, 473-482.

32. Benekli, M., Xia, Z., Donohue, K. A., Ford, L. A., Pixley, L. A., Baer, M. R. et al. (2002). Constitutive activity of signal transducer and activator of transcription 3 protein in acute myeloid leukemia blasts is associated with short disease-free survival. Blood, 99, 252-257.

33. Gordon, J. A. (1991). Use of vanadate as proteinphosphotyrosine phosphatase inhibitor. Methods Enzymol. 201, 477-482.

34. Reed, J. C., Nowell, P. C. \& Hoover, R. G. (1985). Regulation of $c$-myc mRNA levels in normal human lymphocytes by modulators of cell proliferation. Proc. Natl Acad. Sci. USA, 82, 4221-4224.

35. Matikainen, S., Sareneva, T., Ronni, T., Lehtonen, A., Koskinen, P. J. \& Julkunen, I. (1999). Interferonalpha activates multiple STAT proteins and upregulates proliferation-associated IL-2Ralpha, c-myc, and pim-1 genes in human T cells. Blood, 93, 1980-1991.

36. Grigorieva, I., Grigoriev, V. G., Rowney, M. K. \& Hoover, R. G. (2000). Regulation of c-myc transcription by interleukin-2 (IL-2). J. Biol. Chem. 275, 7343-7350.

37. Zhu, M., John, S., Berg, M. \& Leonard, W. (1999). Functional association of Nmi with Stat5 and Stat1 in IL-2- and IFNgamma-mediated signaling. Cell, 96, $121-130$.

38. Ivanov, V. N., Bhoumik, A., Krasilnikov, M., Raz, R., Owen-Schaub, L. B., Levy, D. et al. (2001). Cooperation between STAT3 and c-jun suppresses Fas transcription. Mol. Cell, 7, 517-528.

39. Rossner, M. J., Dorr, J., Gass, P., Schwab, M. H. \& Nave, K. A. (1997). SHARPs: mammalian enhancerof-split- and hairy-related proteins coupled to neuronal stimulation. Mol. Cell. Neurosci. 9, 460-475.

40. Garriga-Canut, M., Roopra, A. \& Buckley, N. J. (2001). The basic helix-loop-helix protein, sharp-1, represses transcription by a histone deacetylasedependent and histone deacetylase-independent mechanism. J. Biol. Chem. 276, 14821-14828.

41. Fujimoto, K., Shen, M., Noshiro, M., Matsubara, K., Shingu, S., Honda, K. et al. (2001). Molecular cloning and characterization of DEC2, a new member of basic helix-loop-helix proteins. Biochem. Biophys. Res. Commun. 280, 164-171.

42. Gingras, S., Simard, J., Groner, B. \& Pfitzner, E. (1999). p300/CBP is required for transcriptional induction by interleukin- 4 and interacts with Stat6. Nucl. Acids Res. 27, 2722-2729.

43. Paulson, M., Pisharody, S., Pan, L., Guadagno, S., Mui, A. L. \& Levy, D. E. (1999). Stat protein transactivation domains recruit p300/CBP through widely divergent sequences. J. Biol. Chem. 274, 25343-25349.

44. Zhang, J. J., Zhao, Y., Chait, B. T., Lathem, W. W., Ritzi, M., Knippers, R. \& Darnell, J. E., Jr (1998). Ser727-dependent recruitment of MCM5 by Stat1alpha in IFN-gamma-induced transcriptional activation. EMBO J. 17, 6963-6971.

45. Giraud, S., Bienvenu, F., Avril, S., Gascan, H., Heery, D. M. \& Coqueret, O. (2002). Functional interaction of STAT3 transcription factor with the coactivator NcoA/SRC1a. J. Biol. Chem. 277, 8004-8011.

46. Nakajima, H., Brindle, P. K., Handa, M. \& Ihle, J. N. (2001). Functional interaction of STAT5 and nuclear receptor co-repressor SMRT: implications in negative regulation of STAT5-dependent transcription. EMBO J. 20, 6836-6844. 
47. Darnell, J. E., Jr (1997). STATs and gene regulation. Science, 277, 1630-1635.

48. Lew, D. J., Decker, T., Strehlow, I. \& Darnell, J. E. (1991). Overlapping elements in the guanylate-binding protein gene promoter mediate transcriptional induction by alpha and gamma interferons. Mol. Cell. Biol. 11, 182-191.

49. Ellenberger, T., Fass, D., Arnaud, M. \& Harrison, S. C. (1994). Crystal structure of transcription factor E47: E-box recognition by a basic region helix-loop-helix dimer. Genes Dev. 8, 970-980.

50. Kiuchi, N., Nakajima, K., Ichiba, M., Fukada, T., Narimatsu, M., Mizuno, K. et al. (1999). STAT3 is required for the gp130-mediated full activation of the c-myc gene. J. Expt. Med. 189, 63-734.

51. Bromberg, J. F. (2001). Activation of STAT proteins and growth control. Bioessays, 23, 161-169. Review.

52. Dinarello, C. A., Novick, D., Puren, A. J., Fantuzzi, G., Shapiro, L., Muhl, H. et al. (1998). Overview of interleukin-18: more than an interferon-gamma inducing factor. J. Leukoc. Biol. 63, 658-664.

53. Miscia, S., Marchisio, M., Grilli, A., Di Valerio, V., Centurione, L., Sabatino, G. et al. (2002). Tumor necrosis factor alpha (TNF-alpha) activates Jak1/ Stat3-Stat5B signaling through TNFR-1 in human B cells. Cell Growth Differ. 13, 13-18.

54. Bromberg, J. F., Horvath, C. M., Besser, D., Lathem,
W. W. \& Darnell, J. E., Jr (1998). Stat3 activation is required for cellular transformation by v-src. Mol. Cell. Biol. 18, 2553-2558.

55. Fukada, T., Hibi, M., Yamanaka, Y., TakahashiTezuka, M., Fujitani, Y., Yamaguchi, T. et al. (1996). Two signals are necessary for cell proliferation induced by a cytokine receptor gp130: involvement of STAT3 in anti-apoptosis. Immunity, 5, 449-460.

56. Chen, R. H., Chang, M. C., Su, Y. H., Tsai, Y. T. \& Kuo, M. L. (1999). Interleukin-6 inhibits transforming growth factor-beta-induced apoptosis through the phosphatidylinositol 3-kinase/Akt and signal transducers and activators of transcription 3 pathways. J. Biol. Chem. 2, 3013-23019.

57. Watanabe-Fukunaga, R., Brannan, C. I., Copeland, N. G., Jenkins, N. A. \& Nagata, S. (1992). Lymphoproliferation disorder in mice explained by defects in Fas antigen that mediates apoptosis. Nature, 356, 314-317.

58. Takahashi, T., Tanaka, M., Brannan, C. I., Jenkins, N. A., Copeland, N. G., Suda, T. \& Nagata, S. (1994). Generalized lymphoproliferative disease in mice, caused by a point mutation in the Fas ligand. Cell, 76, 969-976.

59. Bromberg, J. F., Wrzeszczynska, M. H., Devgan, G., Zhao, Y., Pestell, R. G., Albanese, C. \& Darnell, J. E., Jr (1999). Stat3 as an oncogene. Cell, 98, 295-303.

Edited by M. Yaniv

(Received 12 January 2004; received in revised form 10 May 2004; accepted 17 May 2004) 